\title{
Operator Matrices on Banach Spaces
}

\author{
Nan Hua, Ning Kang, Huan Liao* \\ Department of Mathematics, Science College, Yanbian University, Yanji, China \\ Email: 1466234129@qq.com, ^2235461557@qq.com
}

How to cite this paper: Hua, N., Kang, N. and Liao, H. (2020) Operator Matrices on Banach Spaces. Open Access Library Journal, 7: e6813.

https://doi.org/10.4236/oalib.1106813

Received: September 11, 2020

Accepted: October 13, 2020

Published: October 16, 2020

Copyright $\odot 2020$ by author(s) and Open Access Library Inc.

This work is licensed under the Creative Commons Attribution International License (CC BY 4.0).

http://creativecommons.org/licenses/by/4.0/

\begin{abstract}
Since nonlinear schur theorem was proposed, it broke the limitation of linear operator matrices. And in this paper we study the summability theory for a class of matrices of nonlinear mapping, and the characterizations of a class of infinite matrix transformations are obtained. These results enrich the results on infinite matrices transformations, and have important meaning for the study of Banach space.
\end{abstract}

\section{Subject Areas}

Functional Analysis

\section{Keywords}

Infinite Matrix, Matrix Transformation, Banach Space

\section{Introduction}

A decisive break in the theory of matrix transformations was in 1950, when Robinson considered the action of infinite matrices of linear operators from a Banach space on sequences of elements of that space [1]. In the past years, many remarkable results [2] [3] [4] were yielded in this direction.

Let $X$ and $Y$ be topological vector spaces, and $\mathcal{F}_{0}(X, Y)=\left\{f \in Y^{X}: f(0)=0\right\}$. For sequence families $\lambda(X) \subseteq X^{\mathbb{N}}$ and $\mu(Y) \subseteq Y^{\mathbb{N}}$, the matrix $\left(T_{i, j}\right) \in$ $(\lambda(X), \mu(Y))$ means that $\sum_{j=1}^{\infty} T_{i j}\left(x_{j}\right)$ converges when $\left(x_{j}\right) \in \lambda(X), i \in \mathbb{N}$ and $\left\{\sum_{j=1}^{\infty} T_{i j}\left(x_{j}\right)\right\}_{i=1}^{\infty} \in \mu(Y)$ for each $\left(x_{j}\right) \in \lambda(X)$.

As usual,

$$
\begin{aligned}
& c_{0}=\left\{\left(x_{j}\right) \subseteq \mathbb{C}: x_{j} \rightarrow 0\right\}, \\
& c_{0}(X)=\left\{\left(x_{j}\right) \in X^{\mathbb{N}}: x_{j} \rightarrow 0\right\},
\end{aligned}
$$

*Corresponding author. 


$$
\begin{gathered}
c(X)=\left\{\left(x_{j}\right) \in X^{\mathbb{N}}: \lim x_{j} \text { exists }\right\} \text { and } \\
l^{\infty}(X)=\left\{\left(x_{j}\right) \in X^{\mathbb{N}}: x_{j} \text { is bounded }\right\} .
\end{gathered}
$$

In 2001, Li Ronglu depicted the nonlinear operator matrices transformation with some restrictive condition on topological vector spaces [5]. In the next year, Li Ronglu gave some clear-cut characterizations of the matrix families $\left(c_{0}(X), l^{\infty}(I, Y)\right)$ and $\left(I^{\infty}(X), l^{\infty}(I, Y)\right)$ consisted of matrices of linear and some nonlinear operators between topological vector spaces [6]. In this paper, we study the summability theory for a class of matrices of nonlinear mapping on Banach space, and discuss the characterization of the matrix classes:

$$
\left(l^{\infty}(X), c(Y)\right),\left(l^{\infty}(X), l^{\infty}(Y)\right),\left(c_{0}(X), c_{0}(Y)\right)\left(c_{0}(X), c_{0}(Y)\right) .
$$

All of the researches enrich the results on infinite matrices transformations, and have important meaning for the study of Banach space.

\section{Preliminaries and Lemmas}

In 1993, nonlinear Schur Theorem was given by Li Ronglu and C. Swartz, and broke the limitations of linear operator matrices.

Theorem A. [7] Let $G$ be an Abelian topological group, $\Omega \neq \varnothing,\left(f_{i j}\right)_{i, j \in \mathbb{N}}$ a matrix in $G^{\Omega}$ such that $f_{i j}\left(w_{0}\right)=0$ for some $w_{0} \in \Omega$ and all $i, j \in \mathbb{N}$. If $\left(f_{i j}\right)_{i, j \in \mathbb{N}} \in\left(\Omega^{\mathbb{N}}, c(G)\right)$ i.e., $\lim _{i} \sum_{j=1}^{\infty} f_{i j}\left(w_{j}\right)$ exists for each $\left\{w_{j}\right\} \subseteq \Omega$, then the series $\sum_{j=1}^{\infty} f_{i j}\left(w_{j}\right)$ converges uniformly with respect to both $i \in \mathbb{N}$ and $\left\{w_{j}\right\} \subseteq \Omega$, and $\lim _{i} f_{i j}(w)$ exists for every $w \in \Omega, j \in \mathbb{N}$. If, in addition, $G$ is sequentially complete, then the converse implication is true.

As a special case, the following theorem is a nice result for the matrix family $\left(l^{\infty}(X), c(Y)\right)$.

Theorem B. [8] Let $X, Y$ be topological vector spaces and $T_{i j}: X \rightarrow Y$ a mapping such that $T_{i j}(0)=0$ for every $i, j \in \mathbb{N}$. If $\left(T_{i j}\right)_{i, j \in \mathbb{N}} \in\left(l^{\infty}(X), c(Y)\right)$, then for every bounded $B \subseteq X$, the series $\sum_{j=1}^{\infty} T_{i j}\left(x_{j}\right)$ converges uniformly with respect to both $\left\{x_{j}\right\} \subseteq B$ and $i \in \mathbb{N}$ and $\lim _{i} T_{i j}(x)$ exists for every $x \in X, j \in \mathbb{N}$. If, in addition, $Y$ is sequentially complete, then the converse implication is true.

Note that theorem B exceeded the restriction of linear operators, and a characterization of $\left(T_{i j}\right)_{i, j \in \mathbb{N}} \in\left(I^{\infty}(X), c(Y)\right)$ was given. For Banach spaces $X, Y$, it is useful to discuss the characterization of a variety of matrix families, where the mapping need not be linear.

As preparation of the proves of the main results, we also need following lemma. 
Lemma [9] $\left(x_{j}\right) \in l^{\infty}(X)$ if and only if $\left(t_{j} x_{j}\right) \in c_{0}(X)$ for all $\left(t_{j}\right) \in c_{0}$.

\section{Main Results}

Unless otherwise noted $X, Y$ below are Banach spaces, and the mapping we studied in this section need not be linear.

Theorem 1. Let $T_{i j} \in \mathcal{F}_{0}(X, Y)$ for all $i, j \in \mathbb{N}$, then $\left(T_{i j}\right)_{i, j \in \mathbb{N}} \in\left(l^{\infty}(X), c(Y)\right)$ if and only if

(i) $\lim _{i} T_{i j}(x)$ exists for all $j \in \mathbb{N}$ and $x \in X$;

(ii) For any $\varepsilon>0, M>0$ there exists $m_{0} \in N$ such that $\left\|\sum_{j=m}^{\infty} T_{i j}\left(x_{j}\right)\right\|<\varepsilon$ for all natural number $m>m_{0}, i \in \mathbb{N}$, and $\left\{x_{j}\right\} \subseteq X$ with $\sup _{j}\left\|x_{j}\right\| \leq M$.

Proof. Necessity of condition (i) and (ii) is easy to prove by the theorem B in Introduction.

Now suppose that (i) and (ii) are hold, and $\left(x_{j}\right) \in l^{\infty}(X)$, then for any $\varepsilon>0$, there exists $m_{0} \in \mathbb{N}$ such that $\left\|\sum_{j=m_{0}+1}^{\infty} T_{i j}\left(x_{j}\right)\right\|<\frac{\varepsilon}{3}$ for all $i \in \mathbb{N}$ by the condition (ii). And because of condition (i) there is $i_{0} \in \mathbb{N}$, such that $\left\|T_{k j}\left(x_{j}\right)-T_{i j}\left(x_{j}\right)\right\|<\frac{\varepsilon}{3 m_{0}}$ for all $k, i>i_{0}$. Hence we have

$$
\left\|\sum_{j=1}^{m_{0}} T_{k j}\left(x_{j}\right)-\sum_{j=1}^{m_{0}} T_{i j}\left(x_{j}\right)\right\| \leq \sum_{j=1}^{m_{0}}\left\|T_{k j}\left(x_{j}\right)-T_{i j}\left(x_{j}\right)\right\|<\frac{\varepsilon}{3}
$$

for all $k, i>i_{0}$. Therefore

$$
\begin{aligned}
& \left\|\sum_{j=1}^{\infty} T_{k j}\left(x_{j}\right)-\sum_{j=1}^{\infty} T_{i j}\left(x_{j}\right)\right\| \\
& =\left\|\sum_{j=1}^{m_{0}} T_{k j}\left(x_{j}\right)-\sum_{j=1}^{m_{0}} T_{i j}\left(x_{j}\right)+\sum_{j=m_{0}+1}^{\infty} T_{k j}\left(x_{j}\right)-\sum_{j=m_{0}+1}^{\infty} T_{i j}\left(x_{j}\right)\right\| \\
& \leq\left\|\sum_{j=1}^{m_{0}} T_{k j}\left(x_{j}\right)-\sum_{j=1}^{m_{0}} T_{i j}\left(x_{j}\right)\right\|+\left\|\sum_{j=m_{0}+1}^{\infty} T_{k j}\left(x_{j}\right)\right\|+\left\|\sum_{j=m_{0}+1}^{\infty} T_{i j}\left(x_{j}\right)\right\| \\
& <\frac{\varepsilon}{3}+\frac{\varepsilon}{3}+\frac{\varepsilon}{3}=\varepsilon
\end{aligned}
$$

So $\left\{\sum_{j=1}^{\infty} T_{i j}\left(x_{j}\right)\right\}_{i=1}^{\infty}$ is a Cauchy sequence in $Y$. Therefore $\left\{\sum_{j=1}^{\infty} T_{i j}\left(x_{j}\right)\right\}_{i=1}^{\infty}$ converges by the completeness of $Y$, and then $\left(T_{i j}\right)_{i, j \in \mathbb{N}} \in\left(l^{\infty}(X), c(Y)\right)$. The sufficiency is proved.

Q.E.D.

Since $c_{0}(Y) \subseteq c(Y)$, we can get the next corollary by the theorem.

Corollary 1. Suppose that $i, j \in \mathbb{N}, T_{i j} \in \mathcal{F}_{0}(X, Y)$, then $\left(T_{i j}\right)_{i, j \in \mathbb{N}} \in\left(l^{\infty}(X)\right.$, $\left.c_{0}(Y)\right)$ if and only if $\lim _{i} T_{i j}(x)=0$ for all $j \in \mathbb{N}, x \in X$, and for any $\varepsilon>0, M>0$, there exists $m_{0} \in \mathbb{N}$ such that $\left\|\sum_{j=m}^{\infty} T_{i j}\left(x_{j}\right)\right\| \leq \varepsilon$ for all 
$m>m_{0}, i \in \mathbb{N}$, and $\left\{x_{j}\right\} \subseteq X$ with $\sup _{j}\left\|x_{j}\right\| \leq M$.

Proof. Necessity is clear by above theorem and the definition of $c_{0}(Y)$.

Conversely, let $\left(x_{j}\right) \in l^{\infty}(X)$, then for any $\varepsilon>0$, there exists $m_{0} \in \mathbb{N}$ such that $\left\|\sum_{j=m_{0}+1}^{\infty} T_{i j}\left(x_{j}\right)\right\|<\frac{\varepsilon}{2}$ for all $i \in \mathbb{N}$. Since $\lim _{i} T_{i j}(x)=0$ for all $j \in \mathbb{N}$ and $x \in X$, there is $i_{0} \in \mathbb{N}$, such that $\left\|T_{i j}\left(x_{j}\right)\right\|<\frac{\varepsilon}{2 m_{0}}$ for all $i>i_{0}$ and $j \in \mathbb{N}$.

Hence we have

$$
\left\|\sum_{j=1}^{\infty} T_{i j}\left(x_{j}\right)-0\right\| \leq\left\|\sum_{j=1}^{m_{0}} T_{i j}\left(x_{j}\right)\right\|+\left\|\sum_{j=m_{0}+1}^{\infty} T_{i j}\left(x_{j}\right)\right\| \leq \frac{\varepsilon}{2}+\frac{\varepsilon}{2}=\varepsilon .
$$

So column $\left\{\sum_{j=1}^{\infty} T_{i j}\left(x_{j}\right)\right\}_{i=1}^{\infty}$ converges to 0 , and then $\left(T_{i j}\right)_{i, j \in \mathbb{N}} \in\left(l^{\infty}(X)\right.$, $\left.c_{0}(Y)\right)$. The sufficiency is proved.

Q.E.D.

Theorem 2. Let $T_{i j} \in \mathcal{F}_{0}(X, Y)$ with respect to $i, j \in \mathbb{N}$, then $\left(T_{i j}\right)_{i, j \in \mathbb{N}} \in$ $\left(l^{\infty}(X), l^{\infty}(Y)\right)$ if and only if

(i) $\sup _{i}\left\|T_{i j}(x)\right\|<+\infty$ for all $j \in \mathbb{N}, x \in X$;

(ii) For any $\varepsilon>0, M>0$ and $t_{i} \in C_{0}$, there exists $m_{0} \in \mathbb{N}$ such that $\left|t_{i}\right|\left\|\sum_{j=m}^{\infty} T_{i j}\left(x_{j}\right)\right\|<\varepsilon$ for all $m>m_{0}, i \in \mathbb{N}$ and $\left\{x_{j}\right\} \subseteq X$ with $\sup _{j}\left\|x_{j}\right\| \leq M$.

Proof. $\Rightarrow$ ) Suppose that $T_{i j} \in \mathcal{F}_{0}(X, Y)$, the condition (i) is clear.

Since $\left\{\sum_{j=1}^{\infty} T_{i j}\left(x_{j}\right)\right\} \in l^{\infty}(Y)$ for every $\left(x_{j}\right) \in l^{\infty}(X),\left\{t_{i} \sum_{j=1}^{\infty} T_{i j}\left(x_{j}\right)\right\} \in c_{0}(Y)$ for every $\left(t_{i}\right) \in c_{0}$ by lemma 1 , that is $\sum_{j=1}^{\infty}\left(t_{i} T_{i j}\right)\left(x_{j}\right) \in c_{0}(Y)$. Hence, for every $\left(t_{i}\right) \in c_{0}$, we have $\left(t_{i} T_{i j}\right)_{i, j \in \mathbb{N}} \in\left(l^{\infty}(X), c_{0}(Y)\right)$. Therefore, by above corollary, for every $\varepsilon>0, M>0$ and $\left(t_{i}\right) \in c_{0}$ there is $m_{0} \in \mathbb{N}$ such that for all $m>m_{0}, \quad i \in \mathbb{N}$, and $\sup _{j}\left\|x_{j}\right\| \leq M$, we have

$$
\left|t_{i}\right|\left\|\sum_{j=m}^{\infty} T_{i j}\left(x_{j}\right)\right\|=\left\|\sum_{j=m}^{\infty} t_{i} T_{i j}\left(x_{j}\right)\right\|<\varepsilon .
$$

condition (ii) is proved.

$\Leftarrow)$ For every $j \in \mathbb{N}, x \in X$, and $\left(t_{i}\right) \in c_{0}$, we have $\lim _{i} t_{i} T_{i j}(x)=0$ by the condition (i). Because of the condition (ii), we have $\left(t_{i} T_{i j}\right)_{i, j \in \mathbb{N}} \in\left(l^{\infty}(X), c_{0}(Y)\right)$ by the Corollary 1 , and then for $\left(x_{j}\right) \in l^{\infty}(X)$, we have $\left\{t_{i} \sum_{j=1}^{\infty} T_{i j}\left(x_{j}\right)\right\}_{i=1}^{\infty}$ $\in c_{0}(Y)$ is hold for every $\left(t_{i}\right) \in c_{0}$. Therefore $\left\{\sum_{j=1}^{\infty} T_{i j}\left(x_{j}\right)\right\}_{i=1}^{\infty} \in l^{\infty}(Y)$ by 
lemma 1 , and then $\left(T_{i j}\right)_{i, j \in \mathbb{N}} \in\left(l^{\infty}(X), l^{\infty}(Y)\right)$.

Q.E.D.

Theorem 3. Let $T_{i j} \in \mathcal{F}_{0}(X, Y)$ with respect to $i, j \in \mathbb{N}$, then $\left(T_{i j}\right)_{i, j \in \mathbb{N}} \in$ $\left(c_{0}(X), c_{0}(Y)\right)$ if and only if

(i) $\lim _{i} T_{i j}(x)=0$ for all $j \in \mathbb{N}$ and $x \in X$;

(ii) For any $\varepsilon>0, M>0$ and $\left(t_{j}\right) \in c_{0}$, there exists $m_{0} \in \mathbb{N}$ such that

$$
\sup _{i \in \mathbb{N}, m>m_{0},\left\|x_{j}\right\| \leq M}\left\|\sum_{j=m}^{\infty} T_{i j}\left(t_{j} x_{j}\right)\right\|<\varepsilon
$$

Proof. For $T_{i j} \in \mathcal{F}_{0}(X, Y)$, since $T_{i j} \circ t_{j}(x)=T_{i j}\left(t_{j} x\right), \quad \lim _{i} T_{i j} \circ t_{j}(x)=$ $\lim _{i} T_{i j}\left(t_{j} x\right)=0$ for any $j \in \mathbb{N}, x \in X$ and $t_{j} \in c_{0}$, by condition (i). So condition (i) and (ii) is equivalent to $\left(T_{i j} \circ t_{j}\right)_{i, j \in \mathbb{N}} \in\left(l^{\infty}(X), c_{0}(Y)\right)$ by corollary 1 .

Suppose that $\left(T_{i j}\right)_{i, j \in \mathbb{N}} \in\left(c_{0}(X), c_{0}(Y)\right)$, then $\left\{\sum_{j=1}^{\infty} T_{i j}\left(x_{j}\right)\right\}_{i=1}^{\infty} \in c_{0}(Y)$ for all $\left(x_{j}\right) \in c_{0}(X)$. By lemma $1,\left\{\sum_{j=1}^{\infty} T_{i j}\left(t_{j} x_{j}\right)\right\}_{i=1}^{\infty} \in c_{0}(Y)$, for all $\left(x_{j}\right) \in l^{\infty}(X)$ and $\left(t_{j}\right) \in c_{0}$. Hence $\left(T_{i j} \circ t_{j}\right)_{i, j \in \mathbb{N}} \in\left(l^{\infty}(X), c_{0}(Y)\right)$.

On the other hand, suppose that $\left(T_{i j} \circ t_{j}\right)_{i, j \in \mathbb{N}} \in\left(l^{\infty}(X), c_{0}(Y)\right)$. For every $\left(x_{j}\right) \in c_{0}(X)$, there exists $\left(t_{j}\right) \in c_{0}$ and $\left(z_{j}\right) \in c_{0}(X) \subset l^{\infty}(X)$, such that $\left(x_{j}\right)=\left(t_{j} z_{j}\right)$, and so $\left\{\sum_{j=1}^{\infty} T_{i j}\left(x_{j}\right)\right\}_{i=1}^{\infty}=\left\{\sum_{j=1}^{\infty}\left(T_{i j} \circ t_{j}\right)\left(z_{j}\right)\right\}_{i=1}^{\infty} \in c_{0}(Y)$. Hence $\left(T_{i j}\right)_{i, j \in \mathbb{N}} \in\left(c_{0}(X), c_{0}(Y)\right)$.

Q.E.D.

Theorem 4. Let $T_{i j} \in \mathcal{F}_{0}(X, Y)$ with respect to $i, j \in \mathbb{N}$, then $\left(T_{i j}\right)_{i, j \in \mathbb{N}} \in$ $\left(c_{0}(X), l^{\infty}(Y)\right)$ if and only if

(i) $\sup _{i}\left\|T_{i j}(x)\right\|<+\infty$ for all $j \in \mathbb{N}$ and $x \in X$;

(ii) For any $\varepsilon>0, M>0$ and $\left(s_{j}\right),\left(t_{j}\right) \in c_{0}$, there exists $m_{0} \in \mathbb{N}$, such that $\left\|s_{j} \sum_{j=m}^{\infty} T_{i j}\left(t_{j} x_{j}\right)\right\|<\varepsilon$ for all $m>m_{0}, i \in \mathbb{N}$ and $\sup _{j}\left\|x_{j}\right\| \leq M$.

Proof. By condition (i), for all $\left(t_{j}\right) \in c_{0}$ and $x \in X$,

$$
\sup _{i}\left\|T_{i j} \circ t_{j}(x)\right\|=\sup _{i}\left\|T_{i j}\left(t_{j}(x)\right)\right\|<+\infty .
$$

By theorem 2, condition (i) and (ii) are equivalent to $\left(T_{i j} \circ t_{j}\right)_{i, j \in \mathbb{N}} \in\left(l^{\infty}(X)\right.$, $\left.l^{\infty}(Y)\right)$. Next, we prove that $\left(T_{i j} \circ t_{j}\right)_{i, j \in \mathbb{N}} \in\left(l^{\infty}(X), l^{\infty}(Y)\right)$ for all $\left(t_{j}\right) \in c_{0}$ is equivalent to $\left(T_{i j}\right)_{i, j \in \mathbb{N}} \in\left(c_{0}(X), l^{\infty}(Y)\right)$.

In fact, If $\left(T_{i j}\right)_{i, j \in \mathbb{N}} \in\left(c_{0}(X), l^{\infty}(Y)\right)$, and let $\left(x_{j}\right) \in l^{\infty}(X)$, then $\sum_{j=1}^{\infty}\left(T_{i j} \circ t_{j}\right)\left(x_{j}\right)=\sum_{j=1}^{\infty} T_{i j}\left(t_{j} x_{j}\right)$. Since $\left(t_{j} x_{j}\right) \in c_{0}(X)$ for all $\left(t_{j}\right) \in c_{0}$ by lemma, 
we have $\left\{\sum_{j=1}^{\infty} T_{i j}\left(t_{j} x_{j}\right)\right\}_{i=1}^{\infty} \in l^{\infty}(Y)$. So $\left(T_{i j} \circ t_{j}\right)_{i, j \in \mathbb{N}} \in\left(l^{\infty}(X), l^{\infty}(Y)\right)$. On the other hand, suppose that $\left(T_{i j} \circ t_{j}\right)_{i, j \in \mathbb{N}} \in\left(l^{\infty}(X), l^{\infty}(Y)\right)$. Since for any $\left(x_{j}\right) \in c_{0}(X)$, there must be $\left(t_{j}\right) \in c_{0}, \quad\left(z_{j}\right) \in c_{0}(X) \subset l^{\infty}(X)$, such that $\left(x_{j}\right)=\left(t_{j} z_{j}\right)$, we have

$$
\left\{\sum_{j=1}^{\infty} T_{i j}\left(x_{j}\right)\right\}_{i=1}^{\infty}=\left\{\sum_{j=1}^{\infty} T_{i j}\left(t_{j} z_{j}\right)\right\}_{i=1}^{\infty}=\left\{\sum_{j=1}^{\infty} T_{i j} \circ t_{j}\left(z_{j}\right)\right\}_{i=1}^{\infty},
$$

and then $\left(T_{i j}\right)_{i, j \in \mathbb{N}} \in\left(c_{0}(X), l^{\infty}(Y)\right)$.

Q.E.D.

\section{Result}

In this paper, we first review the research history of infinite matrix transformation, and then we mainly study the summability of a class of nonlinear mapping matrices in Banach space.

And some new results about, matrix transformation theorems are obtained: we characterize the matrix classes such as $\left(l^{\infty}(X), c(Y)\right),\left(I^{\infty}(X), l^{\infty}(Y)\right)$, $\left(c_{0}(X), c_{0}(Y)\right),\left(c_{0}(X), l^{\infty}(Y)\right)$.

\section{Acknowledgements}

This research was supported by the Science and Technology Project of Jilin Provincial Department of Education (JJKH20180891KJ).

\section{Conflicts of Interest}

The authors declare no conflicts of interest regarding the publication of this paper.

\section{References}

[1] Robinson, A. (1985) On Functional Transformations and Summability. Proceedings of the London Mathematical Society, 52, 132-160. https://doi.org/10.1112/plms/s2-52.2.132

[2] Jeribi, A. (2015) Spectral Theory and Applications of Linear Operators and Block Operator Matrices. Springer International Publishing Switzerland. https://doi.org/10.1007/978-3-319-17566-9

[3] Bani-Domi, W. and Kittaneh, F. (2008) Norm Equalities and Inequalities for Operator Matrices. Linear Algebra and Its Applications, 429, 57-67. https://doi.org/10.1016/j.laa.2008.02.004

[4] Bani-Ahmad, F.A. and Bani-Domi, W. (2016) New Norm Equalities and Inequalities for Operator Matrices. Journal of Inequalities and Applications, 2016, Article number: 175. https://doi.org/10.1186/s13660-016-1108-y

[5] Li, R.L., Li, L.S. and Shin, M.K. (2001) Summability Results for Operator Matrices on Topological Vector Spaces. Science in China, 44, 1300-1311. https://doi.org/10.1007/BF02877019

[6] Li, R.L., Kang, S.M. and Swartz, C. (2002) Operator Matrices on Topological Vector 
Spaces. Journal of Mathematical Analysis and Applications, 274, 645-658. https://doi.org/10.1016/S0022-247X(02)00322-0

[7] Li, R.L. and Swartz, C. (1993) A Nonlinear Schur Theorem. Acta Scientiarum Mathematicarum, 58, 497-508.

[8] Maddox, I.J. (1980) Infinite Matrices of Operators. In: Lecture Notes in Math., Vol. 786, Springer-Verlag, Berlin. https://doi.org/10.1007/BFb0088196

[9] Swartz, C. (1978) Applications of the Mikusinski Diagonal Theorem. Bull. Acad. Polon. Sci. Sér. Sci. Math. Astronom. Phys, 26, 421-424. 